\title{
Paiement Pour Services Ecosystemiques, Instrument D'incitation Au Developpement Des Jardins Publics Et Prives Dans Les Communes De Cocody Et Du Plateau (Abidjan - Côte d'Ivoire)
}

\author{
Yao Jean-Clovis Kouadio, Assistant
}

Laboratoire de Botanique, UFR Biosciences, Université Félix Houphouët-Boigny, Côte d'Ivoire, Abidjan, Côte d'Ivoire

Djakalia Ouattara, Maître de Conférences Marie-Solange Tiébré, Maître de Conférences

Laboratoire de Botanique, UFR Biosciences, Université Félix Houphouët-Boigny, Abidjan, Côte d'Ivoire

Centre National de Floristique, UFR Biosciences, Université Félix Houphouët-Boigny, Côte d'Ivoire, Abidjan

Monssou Eugène Oulaïtar, Docteur Richard Nomel, Doctorant

Laboratoire de Botanique, UFR Biosciences, Université Félix Houphouët-Boigny, Côte d'Ivoire, Abidjan, Côte d'Ivoire Kouakou Edouard N'Guessan, Professeur Titulaire

Laboratoire de Botanique, UFR Biosciences, Université Félix Houphouët-Boigny, Côte d'Ivoire, Abidjan, Côte d'Ivoire

Doi:10.19044/esj.2018.v14n26p112 URL:http://dx.doi.org/10.19044/esj.2018.v14n26p112

\begin{abstract}
The public and private green spaces of the communes of Cocody and Plateau of Abidjan city are deteriorating because of lack of financial means for their maintenance. The overall objective of this study is to demonstrate the importance of payments for ecosystem services (PES) in their self-financing. To carry out this study, a survey was conducted among users of 3 public gardens in the Plateau and private garden owners in Cocody. It aimed to identify the willingness to pay (CAP) of users of public gardens for their management and to identify incentives for the creation of private gardens in their own concessions. Analysis of the data reveals a PAC in the form of visiting tax or voluntary donation ranging from 100 FCFA $(0.15 €)$ to 2000 FCFA (3.07 €). Respondents who declare a visiting tax between 100 FCFA and 500 FCFA are the most numerous with a percentage of 92.95. Students, students and unemployed visitors are on the sidelines of visitors who refuse to
\end{abstract}


pay for the management of public gardens. If we stick to the visit fees proposed by the majority of the interviewees, it would be possible to collect 79,800 FCFA (122.76 €) per day for 339,000 FCFA (521.53€) for all 3 public gardens. With regard to the creation of private gardens, a financial subsidy mechanism could motivate the population. This study demonstrates that the introduction of payments for ecosystem services could be an incentive for the development of green spaces in urban areas.

Keywords: Payment, ecosystem services, Abidjan, Côte d'Ivoire

\section{Résumé}

Les espaces verts publics et privés des communes de Cocody et du Plateau de la ville d'Abidjan sont dans un état de dégradation avancée à cause du manque de moyen financier pour leur entretien. La présente étude a pour objectif général de montrer l'importance des Paiement pour Services Ecosystémiques (PSE) dans leur autofinancement. Pour mener à bien l'étude, une enquête a été réalisée auprès des usagers de 3 jardins publics du Plateau et des propriétaires de jardins privés de Cocody. Elle visait à identifier les consentements à payer (CAP) des usagers de jardins publics à la gestion de ceux-ci et à déceler les mesures d'incitation à la création de jardins privés dans leurs propres concessions. L'analyse des données révèle un CAP par voie de taxe de visite ou don volontaire allant de $100 \operatorname{FCFA}(0,15 €)$ à 2000 FCFA $(3,07 €)$. Les enquêtés qui déclarent une taxe de visite entre 100 FCFA et 500 FCFA sont les plus nombreux avec un pourcentage de 92,95. Les élèves, les étudiants et les visiteurs sans emploi constituent la frange de visiteurs qui refusent de payer pour la gestion des jardins publics. Si on s'en tient à la taxe de visite proposée par la majorité des interviewés, il serait possible de récolter par jour $79800 \mathrm{FCFA}(122,76 €)$ à $339000 \mathrm{FCFA}(521,53 €)$ pour 1'ensemble des 3 jardins publics. En ce qui concerne la création de jardins privés, un mécanisme de subvention financière pourrait motiver les populations. Cette étude démontre que l'instauration de paiement pour services écosystémiques pourrait constituer un instrument d'incitation au développement d'espaces verts en milieu urbain.

Mots clés : Paiement, services écosystémiques, Abidjan, Côte d'Ivoire

\section{Introduction}

Le développement des villes durables, plus pourvoyeuses de bien-être humain a contribué au développement de surfaces vertes par la création de parcs nationaux urbains, jardins aménagés, plantations d'alignement, squares, jardins botaniques, aires de jeux mais aussi des pelouses. Il s'est agi sous cet angle de réduire les dégradations écologiques. Ces espaces verts urbains sont 
considérés comme des territoires forestiers et constituent aujourd'hui de véritables zones refuges de la biodiversité (Morsink et al., 1989). La plupart des travaux scientifiques reconnaissent en eux de nombreux services (Arrif $e t$ al., 2011) classés sous le thème de services écosystémiques (MEA, 2005). En effet, la végétation urbaine est à l'origine d'un certain nombre de services rendus à la société et notamment aux citadins qui sont les services d'approvisionnement, les services socioculturels et les services de régulation (MEA, 2005). Les services d'approvisionnement désignent l'approvisionnement ou prélèvement en termes de nourriture (fruits, légumes, feuilles, fleurs...) ou de produits primaires comme le bois énergie, le bois d'œuvre (Oueslati et al., 2006). Ces biens peuvent être autoconsommés, troqués, ou faire l'objet d'un marché. Quant aux services socioculturels, des études récentes réalisées aux Pays-Bas, montrent que les parcs et jardins urbains favorisent la cohésion sociale et territoriale à partir de la construction d'un espace commun et partagé (Peters et al., 2010). Ce sont des espaces de rencontre et de cohabitation qui permettent de renforcer les liens sociaux (Dunnet et al., 2002). Ce rôle est particulièrement souligné pour les jardins partagés (Demailly, 2012a). Ainsi, comme le suggère Llewelyn-Davies (2007) et Demailly (2012b), la prise en compte des infrastructures vertes dans la conception urbaine permet d'atteindre son objectif fondamental qui est celui de créer un espace commun, support du vivre ensemble. Les espaces verts urbains ont également une influence positive sur le niveau d'activité physique des habitants des villes (Croucher et al., 2007). Ce sont des lieux de promenade et de pratiques sportives de plein air. Enfin, le service de régulation est assuré par l'élimination de la pollution atmosphérique (Nowak et Crane, 2002) à travers l'améliore de la qualité locale de l'air. Elle limite la concentration de différents gaz (dioxyde de carbone, oxyde d'azote, ammoniac, dioxyde de soufre...) ou de particules fines (Powe et Willis, 2004 ; Nowak et al., 2007) dans l'atmosphère. Par ailleurs, le processus photosynthétique est responsable de la séquestration du dioxyde de carbone.

En Côte d'Ivoire, dans la ville d'Abidjan, les espaces verts couvraient une superficie de 0,3 $\mathrm{m}^{2} /$ habitant en 1981 (BNETD, 1981). Les arbres y occupent des habitats divers que sont les abords de rue, les cours des habitations, les parcs (le Parc National du Banco), les jardins publics et privés (les jardins publics du Plateau, le jardin botanique du Centre National de Floristique, le jardin botanique de l'Unité de Formation et de Recherche Biosciences, le jardin botanique de Bingerville, la réserve naturelle partielle de Dahlia fleur ...), les espaces délaissés et les plantations intra-urbaines. Malheureusement, certains espaces verts sont soumis à d'intenses pressions anthropiques du fait des aménagements, de l'extension et de la construction des routes, des effets conjugués de l'urbanisation incontrôlée et de l'agriculture urbaine et périurbaine (Nassa, 2009 ; Oura, 2012 ; Akionla, 
2012). D'autres sont aujourd'hui fortement dégradés par manque de moyens pour leur entretien. Par ailleurs, on note au niveau de la population, un manque d'engouement à la création d'espaces verts privés contribuant ainsi au recul du végétal dans les seuls espaces protégés de la ville d'Abidjan que sont le Parc National du Banco et la Réserve naturelle partielle de dalhiafleur.

Cette situation préjudiciable à l'environnement urbain pourrait être atténuée si le manque de financement constituant un frein au développement d'espaces verts à Abidjan était levé à travers un mécanisme d'autofinancement durable. C'est dans cette optique que nous orientons nos recherches. Notre objectif est de montrer l'importance des Paiement pour Services Ecosystémiques (PSE) dans l'autofinancement des espaces verts publics et privés.

De façon spécifique, il s'est agi d'évaluer le consentement à payer (CAP) des visiteurs des trois (3) jardins publics de la commune du Plateau faisant l'objet d'un usage récréatif, pour leur gestion, d'estimer les devises rapportées et enfin de déceler le moyen d'incitation à la création de jardins privés.

\section{MATERIEL ET METHODES}

\section{Zone d'étude}

La présente étude a été réalisée dans les communes du Plateau et de Cocody de la ville d'Abidjan (Figure 1). Abidjan est la principale ville de la Côte d'Ivoire, située dans la région des lagunes au sud-est. Elle s'étend sur une superficie de 57735 ha et comptait en 2014, 6351086 habitants selon l'Institut National des Statistiques (INS). La commune du Plateau s'étend sur une superficie de 250 ha. C'est une commune presqu'île, entourée par la lagune Ébrié. Elle représente le centre administratif, commercial et financier de la Côte d'Ivoire (Diabaté et Kono, 1991). La commune de Cocody s'étend sur une superficie de 13200 ha. Elle est réputée pour ses quartiers résidentiels. Ces deux communes abritent plusieurs espaces verts dont les 3 jardins publics du Plateau (jardin public Briand, jardin public Bressoles et le jardin public du tribunal) qui ont fait l'objet de notre étude et les jardins privés de Cocody. 


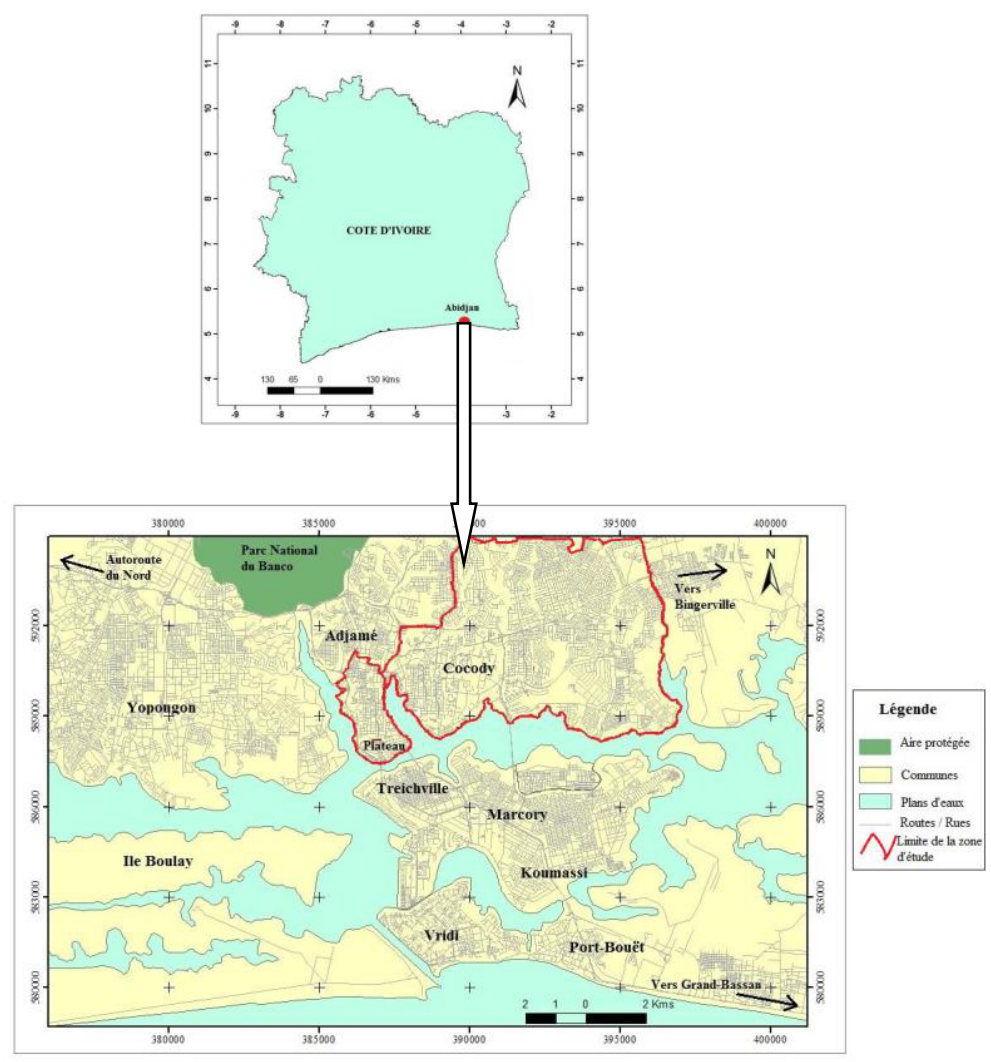

Figure 2: Situation géographique de la zone d'étude

\section{Collecte des données}

Pour mieux appréhender l'intérêt des citadins pour la végétation en milieu urbain, nous avons parcouru les jardins publics et privés pour recueillir respectivement l'avis des visiteurs et propriétaires à travers une enquête. Sur les différents sites d'étude, les enquêtes visaient dans un premier temps à établir le profil des visiteurs de jardins publics et celui des propriétaires de jardins privés. Dans un second temps, il s'est agi d'une part d'identifier les contributions financières des usagers de jardins publics à la gestion de ceuxci et d'autres part de déceler les mesures d'incitation à la création de jardins privés.

Le choix de ces 3 jardins publics de la commune du Plateau réside dans le fait qu'ils sont les plus fréquentés par les populations dans le cadre de leurs activités récréatives.

Le questionnaire a été soumis individuellement aux visiteurs de jardins publics et propriétaires de jardins privés sur les différents sites. Les questions concernant le profil des usagers des jardins publics et propriétaires de jardins privés portaient sur plusieurs critères. Ce sont l'âge, le niveau d'instruction, la profession et le domaine d'activités. En plus de ces quatre critères, les visiteurs 
de jardins publics devraient renseigner les horaires de visite, la fréquence de leur présence, les raisons de visite, le revenu mensuel, le consentement à payer (CAP) pour le service rendu par le jardin public et le moyen d'incitation à la création de jardins privés dans leurs propres concessions.

A l'opposé les propriétaires de jardins privés devraient renseigner en plus du profil, le mode d'obtention des plants qui ont servi à la création de leurs jardins privés.

Au total 297 personnes dont 169 visiteurs de jardins publics et 128 propriétaires de jardins privés ont participé à cette enquête.

Parallèlement aux interviews, nous avons procédé au décompte des visiteurs des jardins publics par dénombrement de tous les visiteurs qui fréquentent chaque site à partir de 8 heures du matin jusqu'à 18 heures. La première heure constitue notre heure d'arrivée sur le site et la seconde, l'heure de retour. Ce dénombrement s'est fait sur 7 jours consécutifs pour tenir compte de l'ensemble des jours de la semaine.

\section{Analyse des données}

Pour l'analyse des données, nous avons procédé dans un premier temps par le test de $\mathrm{Khi}^{2}$ pour comparer les proportions des éléments de réponses recueillies sur la question de savoir ce qui pourrait motiver les populations à créer des jardins privés. La signification du test est déterminée en comparant la probabilité $P$ associée à la statistique du test au seuil $\alpha=0,05$. Par la suite, nous avons complété le test de Khi-2 par une comparaison multiple de proportions par paire avec la procédure de Marascuillo. Ce dernier test a permis de discriminer les proportions pour lesquelles il existe une différence. Dans un second temps, deux types de techniques d'analyse multivariée ont été utilisés pour vérifier les relations entre les variables. Il s'agit de l'Analyse Factorielle des Correspondances (AFC) et de l'Analyse Factorielle Multiple (AFM). L'AFC a été utilisée pour mettre en évidence la relation entre le domaine d'activité et la taxe de visite proposé. L'AFM quant à elle a été utilisée pour montrer les relations entre le profil des usagers de jardins publics et la taxe de visite proposée. Le logiciel $\mathrm{R}$ version 3.1 .3 a été utilisé pour la réalisation de ces tests statistiques.

\section{RESULTATS}

\section{Avis des visiteurs sur la contribution financière et CAP proposé}

Les visiteurs des jardins publics qui consentent à payer une contribution sont estimés à $79 \%$. Cette contribution est exprimée en taxe de visite $(55 \%)$ et don volontaire $(24 \%)$. A l'opposé, ceux qui refusent une contribution financière pour l'entretien des jardins publics sont estimés à $21 \%$.

Les Consentements à Payer (CAP) proposés pour les taxes de visite lors de l'enquête varient de 100 FCFA à plus de 2000 FCFA. Les visiteurs qui 
proposent entre 100 et 500 FCFA sont les plus nombreux avec une proportion de $92,95 \%$ (Figure 2). Ils sont suivis de ceux qui proposent entre 1500 et 2000 FCFA. La proportion de cette catégorie de visiteurs est de 5,28 p.c. La proportion des visiteurs qui pourraient payer entre 1000 et 1500 FCFA et plus de 2000 FCFA est nulle.

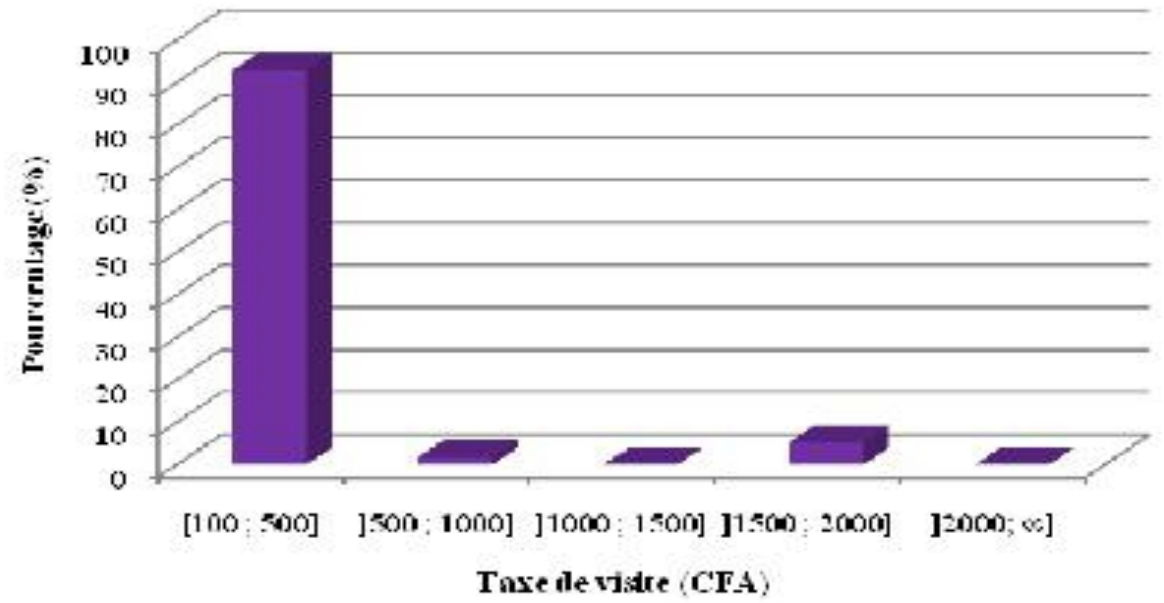

Figure 3: Histogramme de distribution des usagers en fonction des taxes de visite proposées

\section{Relations entre le domaine d'activité et la taxe de visite proposée}

Il existe un lien statistique entre le domaine d'activité et la taxe de visite propose qui représente le consentement à payer (CAP). Les deux premiers axes de l'AFC restituent $30,76 \%$ de la variance des relations entre le domaine d'activité des usagers et le CAP proposé (Figure 3). Le premier axe décrit dans le plan positif, les domaines d'activité tels que l'éducation, l'environnement et la foresterie. Il décrit du côté négatif, le domaine des affaires et plusieurs autres domaines d'activité regroupés sous le vocable « autres ». Le CAP qui décrit au mieux cet axe est celui allant de 1500 à 2000 FCFA. L'axe 2 oppose les domaines d'activité agronomie, TIC, lettre et administration qui sont corrélés positivement contre le domaine des affaires, l'économie et le droit corrélés négativement. La classification des individus décrits par les deux variables qualitatives indique trois groupes de visiteurs. Le premier et le deuxième groupe sont caractérisés par le deuxième axe factoriel tandis que le troisième groupe est caractérisé par le premier axe factoriel. Les visiteurs du groupe 1 sont uniquement ceux du domaine des affaires (soit $39 \%$ de l'ensemble des individus exerçant dans le domaine des affaires) et ceux de divers autres domaines (soit $41 \%$ de l'ensemble des individus de cette catégorie). Tous les visiteurs proposant un CAP compris entre 100 et 500 FCFA appartiennent à ce groupe. Les visiteurs du groupe 2 sont composés de 
$35 \%$ de ceux exerçant dans le domaine des TIC, $35 \%$ de ceux du domaine de l'administration et $23 \%$ de ceux dont le domaine d'activité est la littérature. Tous les individus de cette classe refusent une contribution financière à la gestion des jardins publics. Les visiteurs du groupe 3 sont représentés par ceux qui proposent un consentement à payer (CAP) allant de 1500 à 2000 FCFA. Dans ce groupe, $75 \%$ des visiteurs exercent dans le domaine de l'éducation.

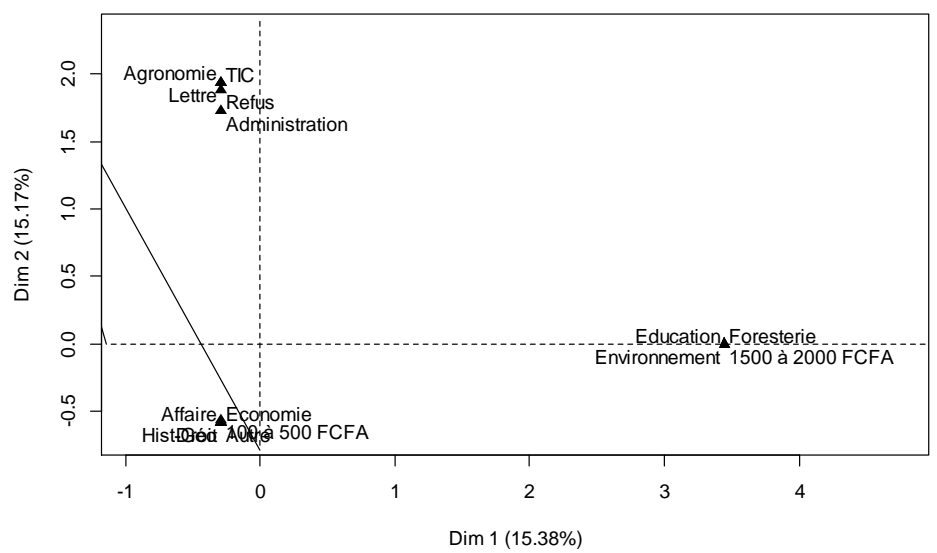

Figure 4: Carte factorielle de distribution des taxes de visite en fonction des domaines d'activité

\section{Relations entre le profil des visiteurs des jardins publics et la taxe de visite proposée}

Les deux premières dimensions restituent $60,75 \%$ de l'inertie totale des relations entre la profession, le revenu et la taxe de visite proposée (CAP). Le revenu mensuel et le CAP contribuent chacun à $33,32 \%$ à la construction de la première dimension tandis que la profession contribue à $33,35 \%$ à sa construction (Tableau 1). Concernant la deuxième dimension, la profession contribue à $52,82 \%$ à sa construction et le CAP à $38,44 \%$. L'examen du premier plan factoriel des variables met en évidence un axe 1 qui oppose les salariés aux sans emplois, étudiants, élèves, commerçants et autres corps de métier. Cet axe décrit du côté négatif une classe 1 regroupant les salariés dont le revenu mensuel varie de 100.000 FCFA à plus de 500.000 FCFA. Leur consentement à payer pour la taxe de visite des jardins publics varie de 500 à 2000 FCFA (Figure 4 et 5). Leurs fréquences de visite sont les plus élevées (de 4 à plus de 5 fois dans la semaine). L'axe 2 oppose une deuxième classe de visiteurs que sont les sans-emplois, les élèves et les étudiants à une troisième classe regroupant les commerçants et tous les autres corps de métier (autres). Les visiteurs au sein de la deuxième classe sont sans revenu et leur fréquence de visite s'élève à 3 fois par semaine. C'est dans cette classe que le refus de la taxe de visite est le plus élevé. Cependant la troisième classe dont le revenu mensuel est limité à 50000 FCFA propose un CAP pour un droit de 
visite allant de 100 FCFA à 500 FCFA. Ces derniers se présentent dans les jardins publics une seule fois par semaine.

Tableau 1: Contribution et qualité de representation des groupes de variables sur les axes factoriels 1 et 2

\begin{tabular}{ccccc}
\hline & \multicolumn{2}{c}{ Axe 1 } & \multicolumn{2}{c}{ Axe 2 } \\
\hline & Contribution & $\operatorname{Cos}^{2}$ & Contribution & Cos $^{2}$ \\
\hline Revenu mensuel & 33,33 & 0,97 & 8,73 & 0,027 \\
\hline $\begin{array}{c}\text { Consentement à } \\
\text { payer (CAP) }\end{array}$ & 33,33 & 0,65 & 38,44 & 0,343 \\
\hline Profession & 33,34 & 0,20 & 52,82 & 0,20 \\
\hline
\end{tabular}

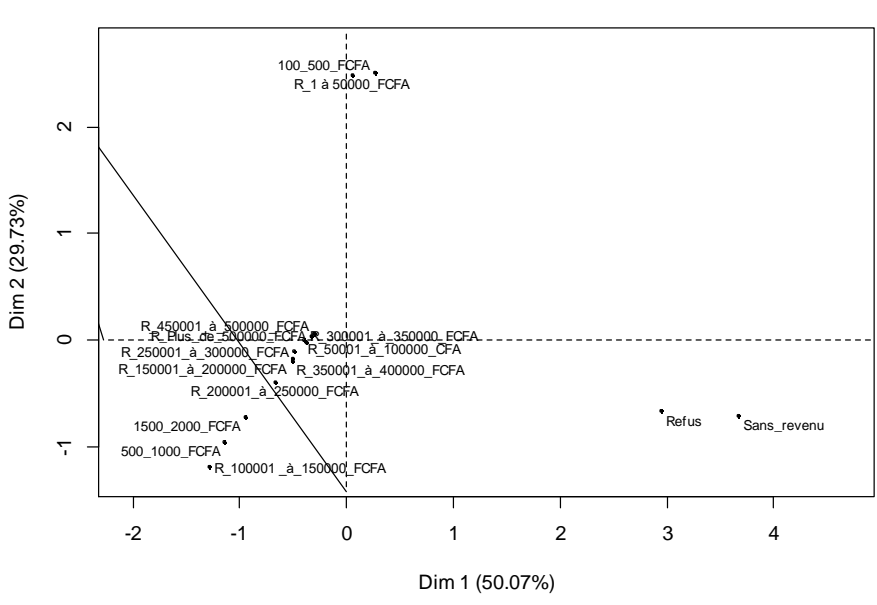

Figure 5: Carte factorielle de distribution des modalités des variables qualitatives utilisées dans $1^{‘} \mathrm{AFM}$

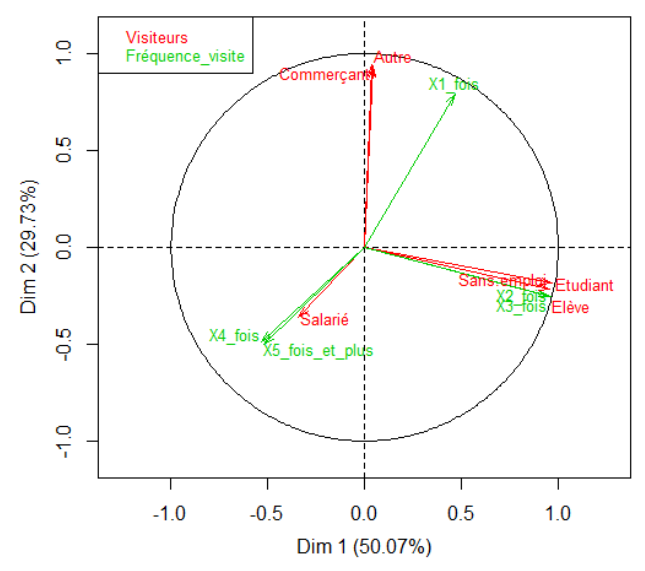

Figure 6: Cercle de corrélation des variables quantitatives utilisées dans l'AFM

\section{Montant éventuel à recueillir après application des PSE}

Le nombre de visiteurs par jour pour les 3 jardins publics s'élève à 798 personnes. Lorsqu'on considère uniquement ceux qui proposent une taxe de visite, on se rend compte que la majorité des personnes $(92,95 \%)$ propose une taxe de visite allant de 100 à 500 FCFA (Tableau 2). Si on s'en tient à cette taxe proposée par la majorité des interviewés, il serait possible de récolter par jour 79800 FCFA à 339000 FCFA. En considérant le nombre de visiteurs par jour (425 visiteurs pour le jardin public Briand, 150 pour le jardin public Bressoles et 223 pour le jardin public du tribunal), la recette en un jour du jardin public Briand varie de 42500 FCFA à 212500 FCFA. Il est suivi du jardin public du tribunal dont la recette variera de $22300 \mathrm{~F}$ CFA à 111500 FCFA. La recette la moins élevée serait enregistrée pour le jardin public Bressoles. Elle varie de 15000 FCFA à 75000 FCFA par jour. 
Tableau 2: Répartition des visiteurs de jardin public en fonction de leur consentement à payer (CAP) proposé

\begin{tabular}{ccc}
\hline Taxe de visite (FCFA) & Pourcentage & Pourcentage cumulé \\
\hline 100 à 500 & 92,95 & 92,95 \\
\hline 500 à 1000 & 1,77 & 94,72 \\
\hline 1000 à 1500 & 0 & 94,72 \\
\hline 1500 à 2000 & 5,28 & 100 \\
\hline Plus de 2000 & 0 & 100 \\
\hline
\end{tabular}

\section{Mode d'obtention des plants des propriétaires de jardins privés}

Le principal mode d'obtention des plants est l'achat. La proportion des enquêtés qui achètent les plants auprès des jardiniers et paysagistes est estimée à 67,64\%. L'obtention par don est cité en second lieu par les enquêtés avec une proportion de $25,29 \%$. La production personnelle des plants est le mode d'obtention qui enregistre la plus faible proportion $(7,07 \%)$.

\section{Moyen d'incitation à la création de jardins privés}

Sur l'ensemble des individus qui se sont prononcés sur ce qui pourraient les motiver ou inciter à créer des jardins privés dans leurs concessions, on constate principalement un engouement pour l'obtention d'une subvention financière $(50 \%)$. La fourniture gratuite des plants $(38,16 \%)$ est citée en second lieu par les populations (Tableau 3). A l'opposé, les enquêtés suscitent peu d'engouement pour la fourniture gratuite de matériel de protection. On enregistre seulement $11,84 \%$ de réponses favorables. Il existe une différence hautement significative entre ces différentes proportions $\left(\chi^{2}=78,27 ; \mathrm{P}<\right.$ $0,001)$.

Tableau 3: Proportion des enquêtés en fonction du moyen d'incitation à la création de jardins privés

\begin{tabular}{cccccc}
\hline & & & & Statistique du test \\
\hline & $\begin{array}{c}\text { Subvention } \\
\text { financière }\end{array}$ & $\begin{array}{c}\text { Fourniture de } \\
\text { plants }\end{array}$ & $\begin{array}{c}\text { Fourniture de } \\
\text { matériel de } \\
\text { protection }\end{array}$ & $\chi^{2}$ & $P$ \\
\hline $\begin{array}{c}\text { Pourcentage des } \\
\text { interviewés }\end{array}$ & 50 & 38,16 & 11,84 & 78,27 & 0,001 \\
\hline
\end{tabular}

\section{Discussion}

La contribution financière à la gestion des jardins publics à usage récréatif de la commune du Plateau trouve l'assentiment de la majorité des usagers. Cependant, les personnes à faible revenu voir sans revenu telles que les étudiants, élèves et sans emplois constituent la frange de la population dans laquelle le refus de payer est le plus élevé. Ce refus pourrait s'expliquer par des moyens financiers insuffisants et l'idée qu'il n'est pas nécessaire de payer pour des biens perçus habituellement comme gratuit. Ces résultats corroborent ceux de Scherrer (2005). Pour Appéré (2004), demander à une personne de se 
prononcer sur une somme monétaire associée à l'amélioration d'un bien qu'elle perçoit habituellement comme gratuit requiert une capacité cognitive extrêmement élevée. Il en résulte un risque des valeurs aberrantes et/ou non cohérentes (par exemple l'existence du biais d'inclusion). Plusieurs autres interprétations sont aussi possibles lorsque le refus de payer est lié à un revenu insuffisant. Il peut s'agir d'un pur comportement de protestation contre l'exercice ou le mode de paiement proposé. Mais, il peut aussi s'agir d'un comportement résultant de la saturation de leur contrainte de revenu. Cette dernière possibilité renvoie elle-même à deux situations. Soit les personnes pourraient procéder à un réaménagement de leurs dépenses pour laisser une place à cette nouvelle dépense, mais ne le souhaitent pas. Leur choix témoigne alors du fait qu'elles accordent une valeur faible ou nulle à l'actif naturel qu'on leur propose d'évaluer. Soit leurs moyens financiers sont effectivement très faibles, et ces personnes ne peuvent pas payer pour autre chose que la satisfaction de leurs besoins immédiats. Parmi elles, existent des personnes qui pourraient accepter de contribuer si leurs revenus augmentaient.

Aussi, ces usagers qui refusent de payer sont certes moins nombreux mais font partie de ceux dont la fréquence de visite est élevée. En ce sens, le refus de payer pourrait s'expliquer par le fait que la taxe de visite leur reviendrait plus cher comparé aux visiteurs qui ne s'y présentent qu'une seule fois dans la semaine.

Plus le revenu mensuel augmente, plus les usagers proposent des CAP plus élevés. Ce constat a été déjà signalé par Scherrer (2005). En effet, dans son étude sur l'évaluation économique des services de récréation du Lac du Der, l'auteur est arrivé à la conclusion que le fait de disposer d'un revenu supérieur accroit la probabilité d'accepter de payer. Le revenu mensuel constitue donc un facteur déterminant dans l'acceptation de payer.

Même si le don volontaire a été moins accepté que la taxe de visite dans la présente étude, certains auteurs comme Amigues et al. (1996) et GomezBaggethum et Barton (2013) estiment que ce mode de paiement permet d'accroître les avis favorables quant à l'instauration des PSE dans les actifs naturels. En effet, le caractère volontaire de cette contribution exerce certainement un effet à la hausse sur l'acceptation de payer. Pour eux, lorsque le support de paiement revêt un caractère obligatoire comme le cas de la taxe de visite, la proportion de personnes déclarant être prêtes à payer diminue. Pour Appéré (2004), l'utilisation de supports de paiment à caractère obligatoire ou volontaire est parfois mal ressentie par les personnes interrogées. En effet, outre le fait qu'on leur demande de payer pour un bien jusque là perçu comme gratuit, ces supports donnent la désagréable impression de leur faire subir le principe peu équitable du pollueur-payeur.

L'instauration du paiement pour services écosystémiques est reconnue aujourd'hui comme un outil devant permettre la gestion durable des 
écosystèmes. Son application devrait générer des devises pour l'entretien d'une part des jardins publics et d'autre part inciter à la création de jardins privés par la population abidjanaise. Les montants qui pourraient être collectés à son application vont de 79800 FCFA (122 €) à 339000 FCFA (521 €) par jour. Pour une période d'un mois, cette valeur varie de 2394000 FCFA (soit $3683 €)$ à 10170000 FCFA (15 646€). Ces montants sont moins élevés que celui qui a été évalué dans le Parc National de la Guadeloupe. Ce dernier varie de $4 €$ (soit 2600 FCFA) à 9000 (soit 5850000 FCFA) par jour). La différence observée pourrait s'expliquer principalement par les niveaux de fréquentation qui seraient très différents et secondairement par la valeur que les communautés accordent aux actifs naturels (Hamadé et al., 2013). En général, la valeur d'un objet réside dans sa rareté. Ainsi, dans les pays à fort couvert végétal, la végétation n'est pas perçue comme un élément rare. Partant de ce constat, en Côte d'Ivoire, les populations accordent moins de valeur à la végétation qu'elles jugent ordinaire. Ces valeurs que l'on pourrait considérer comme la valeur économique des services rendus par les trois jardins publics du Plateau peuvent être discutable sur un certain nombre de points. D'abord, cette estimation ne pourrait représenter la valeur économique totale des services rendus par les trois jardins publics. Pour les auteurs Krutilla (1967), More et al. (1988), Carson et al. (1999), aucune méthode d'estimation de la valeur économique d'un actif naturel ne pourrait fournir à elle seule une valeur économique totale (VET). C'est pourquoi pour y arriver, il importe d'associer plusieurs méthodes. L'intérêt de combiner plusieurs méthodes a déjà été montré par Appéré (2004). Il permet d'une part de tendre vers la réalité dans l'évaluation économique des actifs naturels et d'autre part de contourner le problème de support de paiement. Enfin, le manque d'entretien réel de ces jardins aurait influencé les taxes de visite accordées par les usagers. Le fait que ce facteur soit important dans l'attribution de la valeur économique des espaces verts à été déjà évoqué par Oueslati et al. (2008). En procédant par la méthode contingente basée sur des scénarios correspondant à des améliorations de la qualité d'un actif naturel par rapport à son état initial, l'auteur a observé une augmentation de la probabilité de proposer un consentement à payer élevé.

L'obstacle au développement de jardins privés dans les communes de Cocody serait dû à la cherté des plants vendus sur le marché, augmentant ainsi le coût de revient de la création de jardins privés. En effet, la cherté des plants vendus sur le marché constituerait un obstacle à la création de jardins privés car la principale source d'approvisionnement en plantules telle que révélé par l'étude est l'achat auprès des jardiniers et paysagistes. C'est pourquoi cet obstacle pourrait être levé si une subvention financière est accordée aux personnes contribuant à l'augmentation de la couverture végétale de la ville d'Abidjan. 
Les paiements pour services écosystémiques (PSE) peuvent constituer un instrument pour découpler le développement des villes et la perte de la végétation urbaine, dans la mesure où ils fournissent une incitation directe au changement de pratiques et à la conservation.

\section{Conclusion}

Au terme de cette étude, il convient de souligner que l'instauration des PSE dans la gestion des espaces verts urbains pourrait constituer une source de devises conséquentes pour leur autofinancement. A cet effet, le CAP par taxe de visite ou don volontaire qui trouve le maximum d'assentiment des populations varie entre $100 \mathrm{FCFA}(0,15 €)$ et $500 \mathrm{FCFA}(0,76 €)$. Les élèves, les étudiants et les visiteurs sans emploi constituent la frange de visiteurs qui refusent de payer pour la gestion des jardins publics. Il serait possible de récolter par jour $79800 \mathrm{FCFA}(122,76 €)$ à $339000 \mathrm{FCFA}(521,53 €)$ pour l'ensemble des 3 jardins publics à usage récréatif de la commune du Plateau. En ce qui concerne la création de jardins privés, un mécanisme de subvention financière à toute personne désireuse de créer un jardin privé constituerait une source de motivation. Cette étude démontre l'intérêt de l'instauration des paiements pour services écosystémiques dans le développement d'espaces verts en milieu urbain.

\section{References:}

1. Akionla, M. A. (2012). Diversité et fonctions des formations végétales dans la ville de Porto-Novo. Mémoire de DESS en Gestion de l'Environnement, Université de Parakou, Bénin, 83 p.

2. Amigues, J. P., Desaigues, B. \& Vuong, Q. H., (1996). L'évaluation contingente : controverses et perspectives. Cahiers d'économie et sociologie rurales 40 : 124-150.

3. Appéré, G., (2004). L'évaluation des actifs à usage récréatif: la méthode contingente des coûts de transport. Revue d'économie régionale \& urbaine $1: 81-106$.

4. Arrif, T., Blanc, N., Clergeau, P. (2011). Trame verte urbaine, un rapport Nature-Urbain en géographie et écologie. Cybergo, European Journal of Geographie $17: 5-18$.

5. BNETD (1981). Plan vert d'Abidjan, rapport préliminaire, Abidjan (Côte d'Ivoire), 65 p.

6. Carson, R. T., Flores, N. E. \& Mitchell, R. C. (1999). The theory and measurement of passive use value. In : Valuing Environmental Preferences: Theory and Practice of the Contingent Valuation Method in the US, EC, and Developing Countries, Oxford University Press, Oxford (USA), $19 \mathrm{p}$. 
7. Croucher, K., Myers, L., Jones, R., Ellaway, A. \& Beck, S. (2007). Health and the Physical Characteristics of Urban Neighbourhoods: A Critical Literature Review. Final Report. Glasgow Centre for Population Health, Glasgow, 75 p.

8. Demailly, K. E. (2012a). Les jardins partagés de l'Est parisien : de nouveaux territoires citoyens? Journées scientifiques de la Société d'Écologie Humaine : Les jardins espaces de vie, de connaissance et de biodiversité, Brest (France), 17 p.

9. Demailly, K. E. (2012b). Les délaissés urbains : supports d'une participation citoyenne constitutive de nouveaux territoires? Le cas des jardins partagés de l'Est parisien, Congrès du GIS, Paris (France), $11 \mathrm{p}$.

10. Diabaté, H. \& Kono, L. (1991). Notre Abidjan “toujours plus haut”, Ivoire Media, Abidjan (Côte d'Ivoire), 140 p.

11. Dunnett, N., Swanwick, C. \& Woolley, H. (2002). Improving urban parks, play areas and open spaces. Urban Research Report, London, $217 \mathrm{p}$.

12. Gomez-Baggethum, E. \& Barton, N. D., (2013). Classifying and valuing ecosystem services for urban planning. Ecological Economics 86 : 235-245.

13. Hamadé, F., Gayral, A. M., Hetier, J. P. \& Ronan, C. (2013). Approche des éléments de valeur du Parc national de la Guadeloupe. PNF et BRLI, Montpellier (France), 25 p.

14. Krutilla, J. (1967). Conservation reconsidered. American Economic Review 57 (4) : 777-786.

15. Llewelyn-Davies, Y. (2007). Urban Design Compendium, 2nd Edition, English Partnerships, London, 125 p.

16. MEA (2005). Écosystems and Human Well-being. Vol 2. Island Press, Washington DC (USA), 917 p.

17. More, T. A., Stevens, T. \& Allen, P. G. (1988). Valuation of urban parks. Landscape and Urban Planning 15 (2) :139-152.

18. Morsink, W. A. G., Ubbens, R. M. U., Pickering, J. R., Perkins, R. G., \& Lewis-Watts, P. A. (1989). An urban forestry strategy for Ontario. The Forestry Chronicle 65 (2) : 97-101.

19. Nassa, D. D. A., (2009). Crise de la nature dans l'agglomération abidjanaise : l'exemple de la colonisation, des espaces verts par l'habitat et les commerces dans la commune de Cocody. halshs 1 : 110.

20. Nowak, D. J. \& Crane, D. E., (2002). Carbon Storage and Sequestration by Urban Trees in the USA. Environmental Pollution 116: 381-389. 
21. Nowak, D. J., Hoehn, R. E., Crane, D. E., Stevens, J. C. \& Walton, J. T. (2007). Assessing urban forest effects and values, San Francisco's urban forest. Newtown Square, Department of Agriculture, Forest Service, $22 \mathrm{p}$.

22. Oueslati, W., Madariaga, N. \& Salani, J. (2008). Evaluation contingente d'aménités paysagères liées à un espace vert urbain. Une application au cas du parc Balzac de la ville d'Angers. Revue d'Etudes en Agriculture et Environnement 87 : 77-99.

23. Oueslati, W., Salanié, J. \& Choumert, J. (2006). Modes d'approvisionnement en végétaux: Enseignement de la modélisation économique. Actes des 11ème Assises du CNVVF, 30 novembre et 1er décembre, Vichy, $11 \mathrm{p}$.

24. Oura, R. K., (2012). Extension urbaine et protection naturelle: La difficile expérience d'Abidjan. VertigO-la revue électronique en sciences de l'environnement 12(2). Consulté le 10 décembre 2015.

25. Peters, K., Elands, B. \& Buijs, A. (2010). Social interactions in urban parks: Stimulating social cohesion? Urban Forestry and Urban Greening 9 : 93-100.

26. Powe, N.A. \& Willis, K. G. (2004). Mortality and morbidity benefits of air pollution $\left(\mathrm{SO}_{2}\right.$ and $\left.\mathrm{PM}_{10}\right)$ absorption attributable to woodland in Britain. Journal of Environmental Management 70 : 119-128.

27. Scherrer, S. (2005). Evaluation économique des aménités récréatives d'une zone humide intérieure : le cas du lac du der. Direction des études économiques et de l'évaluation environnementale, France, 75 p. 